Thomas Gloning (Gießen)

Neue mediale Formate und ihre kommunikative Nutzung in der Wissenschaft. Fallbeispiele und sieben Thesen zum Praktiken-Konzept, seiner Reichweite und seinen Konkurrenten

\begin{abstract}
Fragen der sprachlichen Praxis sind in der Sprachwissenschaft seit langem, in vielerlei Gestalt, in Bezug auf ganz unterschiedliche Kommunikationsbereiche und in einer bunten Vielfalt theoretischer Ansätze fest verankert. Im vorliegenden Beitrag diskutiere ich von einem handlungstheoretischen Standpunkt aus, der auch sprachhistorisch-evolutionäre und mediale Erweiterungen umfasst, Vorschläge zur Rolle des Praktiken-Begriffs bzw. (im weiteren Sinne) des Praktiken-Konzepts. Ich formuliere zunächst sieben mehr oder weniger kritische Thesen zu einem Ausschnitt der Praktiken-Literatur, den ich studiert habe, suche dabei auch nach Konsens und Dissens. In ausgewählten Fallbeispielen stelle ich dann Befunde zu Veränderungen in der Wissenschaftskommunikation seit der Erfindung des Buchdrucks dar und frage dabei, ob handlungstheoretisch orientierte Beschreibungen durch die Anwendung des Praktiken-Begriffs gewonnen hätten. Das Resultat fällt eher skeptisch aus.
\end{abstract}

\title{
1 Einleitung
}

Die Herausgeber dieses Bandes haben den „Praktiken“-Begriff bzw. die Frage nach unterschiedlichen „Praktiken“-Begriffen ins thematische Zentrum gestellt und die Beiträger/innen im Vorfeld der Jahrestagung beauftragt, die Reichweite des Praktiken-Konzepts bzw. unterschiedlicher Praktiken-Konzepte für die Sprachwissenschaft $\mathrm{zu}$ diskutieren, Anwendungsperspektiven auszuloten und auch Fragen der Theoriekonkurrenz mit anderen linguistischen Grundbegriffen und mit darauf bezogenen Beschreibungs- und Analyseumgebungen darzulegen (vgl. auch Deppermann/Feilke/Linke in diesem Band). Für die Bewältigung dieser Aufgabe werde ich einen doppelten Weg einschlagen.

(i) Zum einen will ich aus einer eigenen Theorie-Perspektive kritische Anmerkungen zur Praktiken-Diskussion machen, zu ihrem sachlichen Gehalt, aber auch zu Aspekten der Diskursdynamik. Die eigene Perspektive ist ein Theoriegeflecht, das sich aus Wittgensteins Sprachspielidee und weiteren Ansät- 
zen der sprachanalytischen Philosophie, aus der linguistischen Handlungstheorie, Ansätzen zu einer Gebrauchstheorie der Bedeutung, des Meinens und des Verstehens, Formen einer integrativen Betrachtung von Kommunikation und Grammatik, spezifischen Entwicklungen zu textuellen und mündlichen Kommunikationsformen und einigen anderen Elementen speist und zusammensetzt. Hinzu kommt die Verlängerung und weitere Fundierung dieser Elemente in sprachhistorischer und sprachgebrauchshistorischer sowie in einer medialen und multimodalen Perspektive. Diese Ansätze und Entwicklungen, denen eine Orientierung an der sprachlichen Praxis seit langer Zeit eigen ist, bilden den Diskussions- und Beurteilungshintergrund.

(ii) Der zweite Zugriff auf die gestellte Aufgabe sind Fallbeispiele aus einem spezifischen Praxisfeld, der älteren und jüngeren Geschichte der Wissenschaftskommunikation in Phasen medialer Umbrüche. Dabei möchte ich zum einen erkunden, ob der/ein Praktiken-Begriff für kommunikations- und mediennutzungsgeschichtliche Entwicklungen dieser Art eine wesentliche Rolle spielen müsste oder könnte. Darüber hinaus möchte ich aber auch fragen, inwiefern sich im Vergleich der Konzeptionen fruchtbare Anregungen für die eine oder die andere Konzeption ergeben.

\section{Der Praktiken-Begriff, seine Reichweite und seine Konkurrenz. Sieben Thesen}

Ich möchte zunächst sieben kritische Thesen zum Praktiken-Begriff, zur aktuellen Diskussion um das Praktiken-Konzept in der Sprachwissenschaft und auch zum Verhältnis bzw. zur Theorienkonkurrenz mit handlungstheoretischen Alternativen formulieren und erläutern.

1. Eine funktionale, evolutionäre und mediensensitiv unterfütterte Theorie der Sprache und des Sprachgebrauchs auf einer handlungstheoretischen Grundlage ${ }^{1}$ ist eine Theorie der kommunikativen Praxis und der in den soziokulturellen Handlungsbereichen genutzten Praktiken.

In einer solchen Theorieumgebung sind wesentliche theoretisch-methodische Elemente angelegt, die man sowohl im Hinblick auf systematische Fragen der

1 Vgl. aus einer durchaus biografisch geprägten Perspektive hierzu die im Literaturverzeichnis aufgeführten Arbeiten u.a. von Bucher, Fritz, Gloning, Heringer, Keller, Muckenhaupt, Schröder und Strecker. 
Theoriebildung als auch im Hinblick auf viele Gesprächsformen, Texttypen, historische und kommunikationsdynamische Entwicklungen benötigt. Hier ist aus meiner Sicht inzwischen ein fortgeschrittener Stand der Theorie- und MethodenEntwicklung sowie der empirischen Beschreibung erreicht. Soweit ich sehen kann, ist das komplexe Geflecht an Grundannahmen und Beschreibungsmitteln bislang nicht an einer Stelle und im Zusammenhang dargestellt. Und vielleicht sind auch in einem handlungstheoretischen Rahmen unterschiedliche Zuschnitte einer Gesamtdarstellung denkbar und erwartbar. Aber diesen Zustand teilt unsere Wissenschaft von der sprachlich-kommunikativen Praxis (im Rahmen eines handlungstheoretischen Zuschnitts) mit anderen Zweigen der Praxistheorie (vgl. Reckwitz 2003, S. 283 f.).

Man kann deshalb mit einem gewissen Recht fragen, was es bei diesem Stand einer fortgeschrittenen Entwicklung bringt, in programmatischer Weise auf die Überlegungen z.B. von Bourdieus „Entwurf einer Theorie der Praxis“ hinzuweisen, die 1972 veröffentlicht wurde und von der es in der deutschen Ausgabe auf Seite 8 heißt: „Dem zweiten Teil, der für die französische Ausgabe 1972 geschrieben wurde, liegen Aufzeichnungen aus den Jahren 1960 bis 1965 zugrunde“. Bourdieus Gegner ist hier u.a. die Saussure'sche Systemlinguistik, gegen die er das Wechselspiel von Sprachsystem und Sprechen als Praxis setzt (2012, S. 151 ff.). Das ist im Kern richtig, aber inzwischen als Programm nicht mehr originell. Unter Stichwörtern wie „Verfestigung“, „Traditionen des Sprechens“ (Schlieben-Lange 1983; Coseriu 1958, S. 25 f.), ,invisible hand“-Prozesse (Keller 1990, 1995), „Sprache als soziale Gestalt“ (Feilke 1996) und anderen sind inzwischen unterschiedliche Auffassungen verbreitet, die den Zusammenhang von verfestigtem, „geronnenem“ Sprachgebrauch als Grundlage für sprachliche Strukturen, die dann ihrerseits wieder die Grundlage für neue Sprachgebräuche sind, anerkennen.

In systematischer Perspektive tut man bei einem inzwischen gut entwickelten Stand der linguistisch-pragmatischen Theoriebildung wohl gut daran, die aktuellen Beiträge und die Klassiker der Praxis-/Praktikentheorie selektiv zu nutzen und im Hinblick auf einzelne Teilfragen und Aufgaben der Konzeptualisierung von sprachlichen bzw. kommunikativen Gegenständen jeweils zu fragen, ob und ggf. wie sie im Hinblick auf den jeweils aktuellen fachsystematischen Diskussionsstand fruchtbar gemacht werden können.

2. Verfechter des Praktiken-Begriffs verengen teilweise die Vielfalt der linguistischen Traditionen und schaffen damit einen Strohmann, den sie dann mit dem Praktiken-Begriff erfolgreich bekämpfen. Das ist vor dem Hintergrund von weit entwickelten pragmatischen, funktionalen, evolutionären, soziolinguistischen usw. Denktraditionen in der Sprachwissenschaft ärgerlich und dämpft den Rezeptionsappetit. 
Alastair Pennycook schreibt in „Language as a Local Practice“(2010, S. 6):

In order to construct itself as a respectable discipline, linguistics had to make an extensive series of exclusions, relegating people, history, society, culture and politics to a role external to languages.

Das Bild, das an solchen Stellen von der Sprachwissenschaft gezeichnet wird, ist unzureichend und verfälscht. Man kann im Gegensatz dazu sagen, dass in ganz unterschiedlichen Konzeptionen der Sprachwissenschaft viele Elemente des Sprachgebrauchs, der sprachlichen Praxis, der sozialen Einbettung des Sprachgebrauchs, der geschichtlichen Entwicklung von Sprachen in ihren sozio-kulturellen Kontexten, Fragen der Etablierung von Strukturen und Gebrauchsweisen usw. inzwischen sehr gut verankert sind und in der Geschichte des sprachwissenschaftlichen Denkens seit langem präsent waren: Hermann Paul (1920), Marcel Cohens „Sociologie du Langage“ (1956), die von Dell Hymes begründete „Ethnography of Speaking“ (Hymes (Hg.) 1964; Bauman/Sherzer (Hg.) 1974), die Rezeption anthropologischer Arbeiten (u.a. Malinowski 1923, 1984; Frake 1961, 1964), Beschreibungen von Handlungsformen mit Labov (1972) oder stärker losgelöst von sozialen Kontexten (Austin 1956/1957), Gebrauchstheorien der Bedeutung (Gloning 1996), das Werk von Daniel Everett $(2005,2012)$ sind nur einige Beispiele.

Die insgesamt wenig befriedigende Darstellung der Vielfalt in der sprachwissenschaftlichen Diskussionslandschaft in manchen Arbeiten der PraktikenLiteratur wird noch deutlicher, wenn man auch einzelne Theorieelemente betrachtet. Konzeptionelle Elemente wie z.B. das sprachliche Handeln, Formen der Sequenzierung des Handelns, der Zusammenhang von grammatisch-lexikalischer Struktur und kommunikativem Handlungspotenzial (Kompositionalität), das gemeinsame Wissen, eine Vielzahl von Konzeptionen im Umkreis kommunikativer Gattungen, Aspekte der sozialen und kulturellen Differenzierung im Sprachgebrauch, Formen der Multimodalität in mündlichen, schriftbasierten oder hybriden Umgebungen, Diskussionen und Erklärungsmodelle zur Dynamik von Formen des Sprachgebrauchs, die Diskussion um die Rolle kommunikativer Maximen usw. haben eine je eigene Theoriegeschichte. „Die“ Linguistik besteht jedenfalls nicht nur aus de Saussure und Chomsky.

3. Die soziologischen Beiträger zur Praktiken-Diskussion haben eine andere „Gefechtslage“ als die Linguisten. In der Soziologie stehen eigene Probleme auf der Tagesordnung, für die der Praktiken-Begriff ggf. gute Dienste leistet, z.B. bei der Vermittlung der Ebenen des Individuellen und übergeordneter sozialer Strukturen. Aber das heißt nicht, dass man den Begriff auch in der Sprachwissenschaft dringend braucht oder dass man verwandte Grundbegriffe und Denkfiguren nicht schon hätte. 
Eine prominente Gruppe von Autoren, die im Positionspapier der Jahrestagung als grundlegend für den Praktiken-Begriff bzw. für praxistheoretische Ansätze vorgeschlagen wurde, sind Soziologen wie z.B. Theodore R. Schatzki (1996, 2001, 2002), Andreas Reckwitz (2003) oder Anthony Giddens (1995). Exemplarisch möchte ich zwei Anmerkungen zu den Arbeiten von Theodore Schatzki machen, die den Wert solcher Arbeiten einerseits unterstreichen, dann aber auch relativieren.

(i) Zunächst kann man sagen, dass die primäre Motivation der drei Beiträge von Schatzki (1996, 2001, 2002) die Frage nach einer soziologischen Konzeption ist, die unterschiedliche Ebenen und Aspekte des Sozialen integriert. Praktiken stellen in dieser Sichtweise einen systematischen Zusammenhang her zwischen der individuellen Perspektive und den überindividuellen gesellschaftlich-sozialen Einheiten: „Both social order and individuality, in other words, result from practices“ (1996, S. 13).

Im Bereich der Sprachtheorie und der Theorie der Sprachentwicklung sind es unter anderem Invisible-Hand-Konzeptionen (Keller 1990), aber auch andere, z.B. stärker regional orientierte Sprachdynamik-Konzeptionen (Schmidt 2010, Kap. 3), die den Zusammenhang von individuellem Handeln, Sprachbiografien in ihren individuellen Zusammenhängen und überindividuellen Strukturen modellieren. Im Hinblick auf das Verhältnis des Individuellen und des Sozialen im Bereich der Sprache und der Kommunikation wird es also unter anderem darum gehen, den Diskussionsstand in den unterschiedlichen praxistheoretischen Ansätzen abzugleichen mit dem Diskussionsstand in genuin sprachwissenschaftlichen Ansätzen.

(ii) Im Hinblick auf die Schriften Ludwig Wittgensteins (1984), die für Schatzkis Konzeption der zentrale Bezugspunkt sind, und ihre Bedeutung für unterschiedliche Theorien des sprachlichen Handelns kann man sagen, dass dieser Rezeptionsstrang auch in der sprachwissenschaftlichen Tradition seit langem sehr gut ausgebaut ist. Wesentliche Teile der Wittgenstein'schen Sprachspielkonzeption, seine Überlegungen zum Meinen und Verstehen, zum Bedeutungsbegriff, zur Rolle von Handlungszusammenhängen usw. wurden auch in der Sprachwissenschaft thematisiert und schon vor der oder parallel zur Rezeption in der Soziologie fruchtbar gemacht.

Für das Buch von Anthony Giddens (1995) und auch für den Praktikenansatz von Hanks (1987, 1996, S. 3), den man im Schnittbereich von Anthropologie und Soziologie ansiedeln kann, gelten im Hinblick auf sprachwissenschaftliche Fragestellungen und Konzeptionalisierungsaufgaben im Jahr 2015 vergleichbare Befunde: Die Werke sind anregend, sie können aber die fachsystematischen Werkzeuge der Sprachwissenschaft in ihrem Zusammenhang nicht ersetzen. 
4. Der Praktiken-Begriff wird derzeit uneinheitlich verwendet. Es fragt sich, in welcher Version er ggf. für die Sprachwissenschaft weiter fruchtbar werden könnte.

Man kann hier zunächst eine Gruppe von sprachwissenschaftlichen Arbeiten nennen, in denen ein Praktik(en)-Begriff halbwegs klar erkennbar und begrifflich bestimmt ist:

Im Beitrag von Fiehler (2003) zum Beispiel sind Praktiken nicht unähnlich den kommunikativen Aufgaben und den Äußerungsformen, die dafür gebraucht werden, konzeptualisiert. Wir befinden uns hier also im Bereich des Zusammenhangs von Kommunikation und Grammatik.

In einem kurz darauf veröffentlichten Beitrag (Fiehler et al. 2004 zu Eigenschaften gesprochener Sprache) werden Praktiken definitorisch aber ganz anders bestimmt, als Textsorten bzw. Gesprächsformen. Daneben gibt es in dem Buch von 2004 aber auch Textstellen, an denen Einheiten als Praktiken bezeichnet werden, die keine Textsorten und auch keine Gesprächsformen, sondern medialtechnische Formate sind. Ein Brief zum Beispiel ist keine Textsorte, sondern ein mediales Format, das funktional ganz unterschiedlich genutzt werden kann. Wir haben also zwei Veröffentlichungen, die im Abstand von einem Jahr erschienen sind, in denen der Praktiken-Begriff ganz unterschiedlich gebraucht wird, in der späteren Veröffentlichung darüber hinaus terminologisch nicht konsistent. Das erleichtert die Diskussion natürlich nicht.

Ein anderer, relativ klar bestimmter Praktiken-Begriff stammt aus der ethnomethodologisch orientierten Konversationsanalyse (vgl. z.B. Heritage 2011; Schegloff 1972, 1997). Hier sind u.a. einzelne Gesprächsverfahren gemeint, die jeweils eine klar bestimmbare Gesprächsfunktion aufweisen, z.B. das oh-prefacing, Verfahren der Gesprächsbeendigung, Verfahren des Sprecherwechsels oder situativ angepasste Ortsangaben. Im Hinblick auf diese Praktiken kann man sagen, dass sie seit langem im Kernbereich der sprachwissenschaftlichen Gesprächsforschung etabliert sind.

In anderen Arbeiten wird der Praktiken-Begriff eher undeutlich, andeutungsartig und diffus in eine pragmatische Richtung deutend verwendet. Manchmal kann man umschreiben: Praktiken sind „Formen des Handelns“, „Aktivitäten“.

Schließlich: Was ist der Unterschied zwischen „Praxis“ und „Praktik(en)“? In einem Beitrag von Robert Niemann lesen wir in einer Fußnote, dass der Autor die Begriffe „gleichbedeutend“ verwendet (Niemann 2015, S. 248 Fußnote 14). Ich bin nicht sicher, ob man diesem Sprachgebrauch für alle Komplexitätsstufen des sprachlichen Handelns - von den Formulierungsmustern bis zu hochkomplexen Kommunikationsformen - folgen sollte. 
Wenn man schließlich den Praxis-/Praktiken-Begriff in einer übergeordneten Weise nutzt, um möglichst viele relevante „Bestimmungsstücke“ des Handelns und seiner Vollzugs- und Verstehensbedingungen in einen Zusammenhang zu bringen, wie dies im Positionspapier von Deppermann/Linke/Feilke (2014/2015) und nun in der Einleitung des Bandes in beeindruckender Breite systematisiert wurde, dann erkennt man andererseits aber auch, dass die einzelnen Aspekte zum Teil klare Wurzeln in Teilbereichen des sprachlichen Handelns haben und zum Teil für andere Bereiche nur eingeschränkt oder auch gar nicht anwendbar sind. Der Aspekt „Vollzugscharakter“, der sich u.a. auf die zeitliche Strukturiertheit von Praktiken bezieht, spielt in Gesprächen eine ganz andere Rolle (vgl. Deppermann 2007, S. 34 f., 46 ff.) als etwa beim Erstellen und Aufstellen eines Warnschilds. Ressourcen wie Gestik, Mimik, körperliche Orientierung und andere Aspekte der Leiblichkeit (u.a. Mondada/Schmitt (Hg.) 2010) wiederum spielen in vielen schriftbasierten Formen der Kommunikation gar keine oder eine allenfalls instrumentelle Rolle (der Finger, der die Taste drückt).

So stellt sich die Frage, ob das Praktiken-Projekt, verstanden als der Versuch, ein allgemeines Tableau für die zusammenhängende Beschreibung von Formen der Verständigung in den ganz unterschiedlichen sozialen, kulturellen und medialen Zusammenhängen zu erstellen, am Ende dann doch wieder bei den spezielleren Theorien (zu den Gesprächen, Texten, multimodalen Kommunikationsangeboten, zur sprachlichen Höflichkeit, zur Fachkommunikation usw.) ankommen wird.

5. Unterschiedliche Praktik(en)-Begriffe und die Positionen einzelner Vertreter/innen bzw. Gewährsleute sind untereinander nur schwer verträglich. Manche Konzeptionen sind darüber hinaus für sprachwissenschaftliche Ziele und Aufgaben nur bedingt oder auch gar nicht anschließbar.

In seinem weit gespannten Überblick über das Feld der Praxistheorien nennt Reckwitz (2003) eine Vielzahl von Autor/innen, bei denen nicht ohne Weiteres klar ist, ob und ggf. wie die jeweils erkennbaren Grundannahmen und das jeweils verwendete Basisvokabular verträglich sind. $\mathrm{Zu}$ den genannten Autor/innen gehören Ludwig Wittgenstein, Martin Heidegger, Judith Butler, Michel Foucault, Gilles Deleuze und andere. Wenn man aus einer handlungstheoretischen und sprachhistorisch unterfütterten Perspektive auf die Autoren- und Konzeptionenliste schaut, dann gibt es zunächst mehr Differenzen als Übereinstimmungen in den Grundannahmen, in der Darstellungsart und auch in den Zielsetzungen.

Man kann natürlich einwenden, dass eine solche Reserve auch für viele andere ,traditionelle Grundbegriffe der Sprachwissenschaft und der Lehre vom sprachlichen Handeln gilt. Das entbindet aber die Teilnehmer/innen an der Prak- 
tiken-Diskussion nicht von der Aufgabe, die einzelnen Traditionslinien in fachsystematischer Hinsicht $\mathrm{zu}$ sichten und zu bewerten. Wenn man dies tut, wird man feststellen, dass die ,brauchbaren' Gewährsleute bereits fest im sprachwissenschaftlichen Quellenraum verankert sind.

6. Der Praktiken-Begriff ebnet schon erreichte Differenzierungen für die ganz unterschiedlichen Aspekte des kommunikativen Handelns, seiner sprachlichen Realisierung, von Graden der Schematisierung, der historischen Verfestigung usw. sowie Klärungen ihres Zusammenhangs ein. Zumindest liegt hier ein Gefahrenpotenzial. Ob der Praktiken-Begriff auch ein spezifisches Integrationspotenzial hat, bleibt abzuwarten.

Nehmen wir versuchsweise an, wir wollten die bisherigen Resultate und begrifflichen Grundlagen der Sprachwissenschaft im Bereich der (historischen) Pragmatik, der Textlinguistik, der Gesprächsforschung, der Sprach- und Verständigungstheorie, der Multimodalitätsforschung usw. im Lichte eines Praktiken-Begriffs „umfrisieren“ und integrieren: Würden dabei nicht auch wesentliche Differenzierungen verloren gehen? Und wenn man sie erhalten wollte: Wäre man dann nicht wieder bei den spezifischeren Diskussionsständen in den ursprünglichen Disziplinen (siehe auch These 4)? Ich denke hierbei etwa an die frühen Arbeiten von Paul Grice (1989) aus den späten 1950er (,Meaning“, 1957) und 1960er Jahren zu Fragen der Bedeutung, des Meinens und des Verstehens. Oder an Differenzierungen und Erklärungsmuster aus dem Bereich der Sprachwandeltheorie und der Historischen Semantik. Oder an Forschungen zu spezifischen Texttypen, Gesprächsformen, medialen Angeboten und ihrer Nutzung, zu bestimmten grammatischen Konstruktionen und ihrer kommunikativen Nutzung, zur Lehre von der Themenorganisation und den Verfahren des Themenmanagements usw.

Ich sehe und teile das Bedürfnis nach einer integrativen Gesamtdarstellung und Organisation all dieser und vieler anderer Aspekte des sprachlichen Handelns bzw. der Verständigungpraxis im weitesten Sinne. Ich sehe aber noch nicht, dass dieses Versprechen in der Praktiken-Literatur bislang eingelöst wurde.

Wenn man die Frage nach dem Zusammenhang und der Zusammenführung unterschiedlicher Aspekte der sprachlichen Praxis stellt, bietet sich als Alternative eine netzwerkartige Darstellung der Grundannahmen und der darauf bezogenen Grundbegriffe an, dabei wäre die wesentliche Grundlage begriffliche und konzeptuelle Vielfalt, die der Vielgestalt des Gegenstandsbereichs besser angemessen ist.

Die Arbeit an einer stärkeren theoretischen Integration von Aspekten des sprachlichen Handelns bzw. der kommunikativen Praxis in ganz unterschiedlichen Bereichen ist jedenfalls eine Aufgabe, die Praktiken-Theorie(n) und die Theorie(n) des sprachlichen Handelns teilen. 
7. Es wäre gut, wenn man zwei Dinge besser auseinanderhalten würde: den einen (wenn auch diffusen) Praktiken-Begriff und die Vielfalt unterschiedlicher praxeologischer bzw. an spezifischen sprachlich-kommunikativen Praxisfeldern bzw. Praxiselementen orientierter Forschungsansätze.

Ein vereinheitlichender Praktiken-Begriff birgt, wie erwähnt, die Gefahr, dass bereits ausgearbeitete Differenzierungen in ganz unterschiedlichen Bereichen der sprachwissenschaftlichen Theoriebildung wieder eingeebnet werden. Die oben in These 6 formulierte Präferenz von Vielfalt und gegenstandsspezifischer Begriffsbildung ist aber dennoch gut verträglich mit einer praxeologischen Perspektive, also einer Forschungsausrichtung, die an den unterschiedlichen Zusammenhängen und den zum Teil spezifischen Bedingungsfaktoren im Rahmen einzelner sprachlich-kommunikativer Praxisfelder und ihrer soziokulturellen Einbettung im weitesten Sinne interessiert ist (vgl. z.B. Streeck 2001, in diesem Band).

Ich wähle als ein Beispiel ${ }^{2}$ und zur Verdeutlichung dieses Punkts die gerade erschienene Arbeit von Anja Stukenbrock (2015) über die Nutzung von Gesten in face-to-face-Interaktion. Die Untersuchung versteht sich programmatisch als eine praxeologische Arbeit (2015, S. 1, 12 f., 493), aber der Begriff der „Praktik“ kommt im ganzen Buch nur viermal vor (ein paar zitierte practice-Belege kommen hinzu), der Ausdruck trägt an den betreffenden Stellen keine theoretische Last, er wird nicht terminologisch eingeführt, er kommt auch im Register nicht vor, verwandte Ausdrücke sind u.a. „Verfahren“, für das Zeigen selbst kommt „Zeigehandlung“ dagegen sehr häufig vor. Der Ausdruck „Praxis“ ist auch eher selten verwendet, zum einen zur Bezugnahme auf die kommunikative Praxis in einem allgemeinen Sinn, sodann für eine Gesprächsform, das „Petzen“, hierfür variierend auch „kommunikative Gattung“, sodann eine Wendung wie „Praxis des Zeigens“, die wiederum mit „Zeigehandlung“ näher verbunden ist. Daneben finden wir eine gewisse Vielfalt von weiteren Begriffen wie „Interaktion“, „Ressource“, „Zeigegeste" usw.

In dieser sehr überzeugenden und ertragreichen Arbeit steht ein spezifischer Bereich der kommunikativen Praxis, die Verfahren, Funktionen, Bedingungen, Typen usw. der Nutzung von Gesten im Zusammenhang mit sprachlichen Äuße-

2 Im Beitrag von Angelika Linke (2011) zur Rolle von Musterbildungen auf unterschiedlichen sprachlichen Beschreibungsebenen in einer kulturgeschichtlichen Perspektive wird der Praktiken-Begriff ebenfalls nur variierend gebraucht. Aus meiner Sicht ist es einleuchtend, z.B. für Aspekte der Kollokationen, der Phraseologismen usw. auch die Unterscheidungen und Beschreibungsmittel zu nutzen, die in den darauf bezogenen Forschungsbereichen speziell entwickelt wurden. 
rungen in face-to-face-Interaktionen im Vordergrund. Der Praktiken-Begriff spielt dabei keine nennenswerte Rolle, der theoretisch-begriffliche Werkzeugkasten umfasst ältere und jüngere Bestandteile gleichermaßen und folgt den spezifischen Anforderungen des Gegenstandsbereichs, ohne dass der praxeologische Charakter der Arbeit dadurch in irgendeiner Weise zweifelhaft würde.

\section{Fallbeispiele: Neue mediale Formate und ihre kommunikative Nutzung in der Wissenschaft}

Ich werde nun einen bestimmten Typ von historischer Entwicklung und seine Beschreibung in den Vordergrund rücken und ausgewählte Veränderungen in der Wissenschaftskommunikation diskutieren, die im Gefolge neuer medialer Möglichkeiten entstanden sind. Diejenigen Theorieelemente und Beschreibungswerkzeuge, die man für die Beschreibung dieser historischen Kommunikationsformen benötigt, sind in einer funktionalen, evolutionär und lebensformorientierten sowie mediensensitiv aufgerüsteten Handlungstheorie schon vorhanden. Ich werde deshalb an einigen Stellen Hinweise geben und Aspekte diskutieren, die für die Praktiken-Diskussion fruchtbar sein könnten, und Konsens und Dissens zwischen den Konzeptionen immerhin andeuten. Ich betrachte diese Art der Beschreibung als eine praxis-orientierte Beschreibung, die aber nicht zentral auf dem Praktiken-Begriff beruht, sondern ihre zentralen Beschreibungswerkzeuge von den Erfordernissen des Gegenstands her auswählt.

\subsection{Ausgangspunkte und Fragestellung}

Wenn neue Medien verfügbar werden, verändern sich die kommunikativen Handlungsspielräume. An Beispielen aus dem Bereich der Wissenschaftskommunikation lässt sich der grundlegende Zusammenhang zwischen der Verfügbarkeit neuer Medien, der Entstehung neuer medial-technischer Formate, der Entstehung von kommunikativen Nutzungspotenzialen der neuen Formate sowie die Frage nach ihren tatsächlichen Nutzungsweisen aufzeigen.

Im Folgenden gebe ich in gebotener Kürze ${ }^{3}$ Fallbeispiele aus Geschichte und Gegenwart der Wissenschaftskommunikation. Die zentrale Fragestellung dabei

3 Die Fallbeispiele werden in einer ausführlicheren Online-Veröffentlichung, die später erscheinen wird, genauer und materialreicher dargestellt und erläutert. 
ist, ob der Praktiken-Begriff für die Analyse von Gegenständen dieser Art eine nennenswerte Rolle spielen kann und welches Anregungspotenzial ggf. für einzelne Aspekte davon ausgeht.

\subsection{Mediale Formate, kommunikative Handlungsspielräume und ihre tatsächliche Nutzung}

Ausgangspunkt für die Frage nach dem Zusammenhang von medialen Formaten, kommunikativen Handlungsspielräumen und der tatsächlichen Nutzung dieser Spielräume sind tiefgreifende Veränderungen im Bereich einer digitalisierten, globalen Medienkommunikation, die auch weite Teile der Wissenschaftskommunikation betreffen und die mit Stichwörtern wie Social software, Web 2.0, Interaktivität, user generated content, participatory culture adressiert wurden.

Digitale Formate sind zunächst nur technisch-medial bedingte Arrangements, die für ganz unterschiedliche funktionale Nutzungen offen sind. Beispiele für solche medial-technischen Arrangements sind etwa: Mailingliste, Blog, Wiki oder (andere Arten von) Webseiten. Formate dieser Art stellen einen technischen Gestaltungsrahmen dar, der im Hinblick auf kommunikative Ziele und die verschiedenen Aspekte der Interaktion sehr unterschiedlich genutzt werden kann.

Formen der kommunikativen Nutzung von medialen Formaten sind von funktionalen Parametern geprägt. Hierzu gehören insbesondere die Funktion(en), die Handlungsstruktur, Aspekte der Themenorganisation und des Themenmanagements, typische Äußerungsformen, spezifische Kommunikationsmaximen, typische Formen des Wissensaufbaus, der Beteiligungsstruktur, usw. Beispiele für etablierte Formen der kommunikativen Nutzung von Formaten in der Wissenschaft sind z.B.: Rezension, Fachaufsatz, fachliche Monografie, Lehrbuch, Handbuch, wissenschaftlicher Nachruf, Call for Papers und mehrere andere.

Vor allem im Bereich neuerer multimodaler Kommunikationsangebote in Printmedien und in digitalen Umgebungen finden sich zahlreiche Angebote, in denen sich der Gebrauch einzelner Ressourcen wie z.B. der Farbe oder des räumlichen Arrangements nicht auf konventionelle oder auch nur mehr oder weniger schwach routinisierte Praktiken zurückführen lässt. Solche neuartigen Verwendungen sind eine wichtige Quelle der Innovation, sie können dann Gegenstand der Selektion und der zunehmenden Verfestigung im Lauf weiterer kommunikativer Nutzungen werden.

Das folgende Beispiel zeigt eine spontane Konstellation aus einem Posting einer Mailingliste, einem Blog-Beitrag, einem systematischen Wiki und einer 
Zotero-Gruppe. Die Funktion der Nutzung einer solchen Formatkonstellation ${ }^{4}$ ist die kollaborative Erstellung einer fachlich spezifischen Literaturübersicht. Ausgangspunkt ist ein Posting an die Humanist-Liste, eine der ältesten Mailinglisten überhaupt und thematisch spezialisiert im Bereich der Computernutzung in den Geisteswissenschaften. Das Posting an die Mailingliste hat folgenden Text:

To: humanist@lists.digitalhumanities.org

Subject: Digital Classics Bibliography

[apologies for cross posting]

Dear Humanists, ... I'm working on a literature review on the theme Classics

and Computers. In order to allow other people with any interest in this to contribute to my initial list I created an open group on Zotero called _digitalclassics_. To find out more please check out my recent blog post http://www. stoa.org/archives/1216. Thanks in advance, Matteo

In diesem Posting wird auf einen ausführlicheren Blog-Beitrag verlinkt. Folgt man diesem Link, dann kann man im Blog-Beitrag folgende Ausführungen lesen:

For the time being, in order to allow anyone with any interest in this to contribute I created a group on Zotero called digitalclassics. The group is open (i.e. my authorisation is not needed to join) so please join it and start contributing your entries to the list. I'm thinking in particular of publications that I have unintentionally neglected and/or publications in other languages that I was not aware of. [...] As soon as the bibliography will reach a reasonably stable shape I will update the page I have already created on the DigitalClassicist wiki.

Insgesamt haben wir es hier also mit einer spontanen, funktional motivierten Verknüpfung von vier unterschiedlichen Formaten $\mathrm{zu}$ tun, die jeweils unterschiedliche Aufgaben im Hinblick auf das übergeordnete Ziel haben. Die Mailingliste und der Blog dienen der Verbreitung des Anliegens und der Gewinnung von Mitarbeiter/innen, die Bookmarking-Gruppe bei Zotero dient der kollaborativen Sammlung von Literaturtiteln, das Wiki dient der systematischen Ergebnisdokumentation.

Im Hinblick auf den Zusammenhang von neuen Medien, neuen medialen Formaten und ihrer kommunikativen Nutzung stellen sich in einer evolutionären Perspektive folgende kommunikations- und mediengeschichtlichen Leitfragen:

4 Zum Begriff, ,Formatkonstellation`vgl. Fritz/Bader (2010, S. 341 ff.). 
a) Welche medialen Formate sind zu einem bestimmten Zeitpunkt (in einer Nutzergemeinschaft/einem Fachgebiet) verfügbar?

b) Wie werden einzelne mediale Formate $\mathrm{zu}$ einem bestimmten Zeitpunkt (in einer Nutzergemeinschaft) kommunikativ genutzt?

c) Wie entwickeln sich Darstellungsformen/Genres, wenn ein neues Format verfügbar ist? Welche Rolle spielen traditionelle Genres?

d) Welche kommunikativen Formen der Interaktivität, der Kollaboration, der Partizipation sind in einem medialen Format vorgesehen, welche sind möglich, welche werden tatsächlich genutzt?

e) Wie ist das Zusammenspiel individueller Handlungen und kollektiver Resultate zu modellieren, z.B. im Hinblick auf die Ausbildung von Netzwerken, von intertextuellen Gefügen oder für die Etablierung neuer Genres?

f) Wie verlaufen und welche Rolle spielen reflexive Medien- und Mediennutzungsdiskussionen ${ }^{5}$

g) Welche Konsequenzen ergeben sich daraus für eine evolutionäre Theorie und die Geschichte der Wissenschaftskommunikation (als Praxis)?

Man sieht an dieser Form der thematischen Exposition und auch an der Formulierung der Leitfragen, dass dabei eine klare Perspektive auf die kommunikative Praxis zugrunde liegt, dass aber nicht der Praktiken-Begriff eine zentrale Rolle spielt, sondern eine Mehrzahl von Grundbegriffen, die sich aus dem handlungstheoretischen und dem medientheoretischen Vokabular speisen.

Ich möchte den Zusammenhang zwischen der Verfügbarkeit neuer Medien bzw. neuer medialer Technologien, neuen medialen Formaten, ihrem Nutzungspotenzial und ihren tatsächlichen Nutzungen anhand von vier historischen Beispielen veranschaulichen.

\subsection{Der Buchdruck bedingt Veränderungen in der Lehr-/ Lern-Kommunikation der frühen Universitäten}

Um die Mitte des 15. Jahrhunderts entwickelte Johannes Gutenberg in Mainz den Druck von Texten mit Hilfe von einzeln kombinierbaren Metall-Lettern. Eine wichtige Folge, die sich aus der Nutzung dieser neuen medialen Technologie er-

5 Wenn neue Medien verfügbar werden, findet häufig eine reflexive Diskussion des Mediums und von Formen seiner Nutzung statt. Die Zeitungsdebatte des 17. Jahrhunderts, Diskussionen um den frühen Film, aber auch Themenstränge wie der Zusammenhang von Gewalt und Fernsehen sind historische Beispiele solcher Formen der Reflexion. 
gab, betraf die Lehr-/Lern-Kommunikation an den frühen Universitäten. In der neuen buchgestützten Lehr-/Lern-Konstellation bedingt die Tatsache, dass die Studenten in der Vorlesung bereits im Besitz der Texte sind, Veränderungen der Handlungsstruktur. Im Mittelpunkt steht nun nicht mehr die Vervielfältigung eines handschriftlich verfügbaren Texts, sondern die Erläuterung eines bereits vorliegenden Texts (Giesecke 1991, S. 217 ff.).

Gegen Ende des 15. Jahrhunderts war die neue Lehrpraxis offenbar etabliert. In den Statuten des Freiburger „Collegium Sapientiae“ (1497), einer Art Studienstiftung, ist die neue Praxis bereits als Regelfall festgeschrieben:

Jeder Inwohner unseres Hauses ızur Weisheit ‘ höre - wenn er nicht durch ein gerechtfertigtes Hindernis aufgehalten ist - seine Vorlesungen, vor allem die gewöhnlichen, sorgfältig und ohne irgendwelche Unterbrechung, mit einem eigenen oder ihm zu bequemlichem Gebrauch gegebenen Buche (ex libro proprio vel commode ad vsum sibi dato); es schöpft nämlich Wasser mit dem Sieb, wer ohne Buch lernen will. (Statuta Collegii Sapientiae 1497 [1957], S. 82 f.; Hervorh. TG).

Auch in die Bildrepräsentation der Freiburger Statuten ist die neue Lehr-/LernKonstellation eingegangen: Den Studenten der in Abbildung 1 wiedergegebenen Magister-cum-discipulis-Szene liegen die Texte vor, die in der Vorlesung behandelt werden. Der Finger, der im Bild auf eine Textstelle im Buch zeigt, ist ein physischer Hinweis auf die Aspekte der Aufmerksamkeit und der textuellen Bewegung während der Vorlesung.

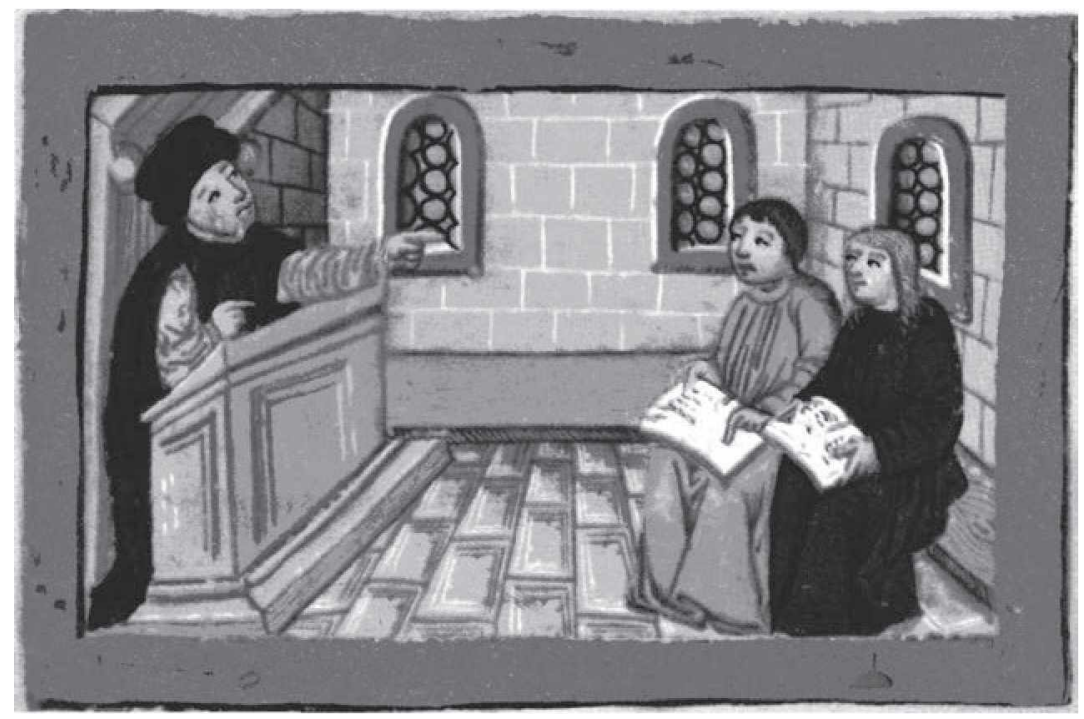

Abb. 1: Magister-cum-discipulis-Szene aus den Freiburger „Statuta Collegii Sapientiae“ (1497 [1957]) 
Die Abbildung des mit dem Finger im Text folgenden Zuhörers deutet darauf hin, dass die Veränderung des kommunikativen Arrangements auch Veränderungen im körperlichen Betragen mit sich gebracht hat, die sehr eng mit Fragen der Aufmerksamkeit, der Aufmerksamkeitssteuerung und möglicherweise auch mit Verfahren der thematischen Bewegung zusammenhingen. Vorlesungen dieser Art sind eine face-to-face-Interaktion, bei denen man die Fragen nach dem körperlichen Benehmen (u.a. der „mitlesende“ Finger, Dynamik der Blickrichtung, Dynamik der körperlichen Ausrichtung) und seiner Rolle für die Organisation der Interaktion mit großem Recht stellen kann. Aus der Praktiken-Perspektive kann man die Frage nach dem veränderten körperlichen Benehmen in den „,neuen“ Vorlesungen vom Ende des 15. Jahrhunderts also mit Recht hervorheben, auch wenn sie sich aufgrund der beschränkten Quellenlage vielleicht nur schwer beantworten lassen wird.

Die wesentlichen Aspekte der Veränderung lassen sich aber mit bereits verfügbaren Beschreibungsmitteln (u.a. Handlungsstruktur, Interaktionsrollen, Themenmanagement) einer sprachwissenschaftlichen Geschichte von Kommunikationsformen analysieren und rekonstruieren.

\subsection{Formen der Textnutzung in wissenschaftlichen Zeitschriften des späten 17. und 18. Jahrhunderts}

Eine weitere grundlegende Veränderung in den Wissenschaften ist mit der Entstehung allgemeiner und fachlich spezialisierter Zeitschriften seit dem späten 17. Jahrhundert und dann vor allem im 18. Jahrhundert verbunden, die Thomas Habel in seiner Studie „Gelehrte Journale und Zeitungen der Aufklärung“ (2007) aufgearbeitet hat.

Im Hinblick auf den Zusammenhang neuer Medien und neuer kommunikativer Möglichkeiten ist vor allem die Entstehung neuer Darstellungsformen, neuer Texttypen hervorzuheben. Eine wichtige Darstellungsform in Zeitschriften dient dazu, eigene neue Forschungsergebnisse in Aufsatzform darzustellen. Hier liegen die Wurzeln des modernen wissenschaftlichen Zeitschriftenartikels, der sogenannten Originalarbeit, zu denen historische Studien z.B. von Ylönen (2001) und Gross/Harmon/Reidy (2002) vorliegen.

Als Beispiel führe ich hier eine Passage aus der Vorrede des von F.A.C. Gren ins Leben gerufenen „Journal der Physik“ (1790 ff.) an. In der programmatischen Vorrede zu dieser neuen Zeitschrift, die damals auch wesentliche Gegenstände dessen, was heute zur Chemie gehört, umfasste, äußert sich Gren auch zum Spektrum der Darstellungsformen, der Texttypen: 
Mein Zweck bey der Herausgabe dieses Journals ist, die Entdeckungen der Aus- und Innländer im mathematischen und chemischen Fache der Naturlehre bekannt zu machen, neuere Lehrmeynungen, neuere Erfahrungen, Beschreibungen und Abbildungen dazu gehöriger Werkzeuge ... mitzutheilen ... Das Werk enthält 1) eigenthümliche Abhandlungen, um Naturforschern Gelegenheit zu geben, ihre Beobachtungen, Entdeckungen und Bemerkungen, die sie zur Förderung der Naturlehre bekannt machen wollen, frühzeitig mittheilen zu können. [...] Da die Anschaffung der Denkschriften von Societäten und Akademien der Wissenschaften dem Privatmanne oft nur gar zu schwer fällt, so hielt ich es für nöthig 2) vollständige Auszüge der physikalischen Abhandlungen der Academien und Societäten der Wissenschaften zu geben; und eben so hoffe ich auch 3) durch die Auszüge und Abhandlungen ausländischer Journale ... die Anschaffung der letztern entbehrlich zu machen. 4) Die litterarischen Anzeigen haben nicht sowohl die Beurtheilung, als die Bekanntmachung der Bücher zum Zweck. (F.A.C. Gren (Hg.), „Journal der Physik“, Bd. 1, 1790, Vorrede)

Man erkennt in dieser Passage des Vorworts zu einer neuen Zeitschrift eine Art von systematischer Planung und Begründung der genutzten Darstellungsformen bzw. Textsorten, die drei funktionale Schwerpunkte abdeckt: (i) Darstellung und Mitteilung eigener, neuer Forschungsergebnisse; (ii) Information über neue wissenschaftliche Publikationen; (iii) Bewertung und Beurteilung neuer wissenschaftlicher Publikationen. Im Jahr 1790 konnte sich Gren bei dieser Profilierung der Darstellungsformen auf bereits etablierte Muster stützen; die „eigenthümlichen Abhandlungen“ sind jedenfalls als eine der zentralen Darstellungsformen erwähnt.

Neben den Rezensionen und den Originalabhandlungen sind Zeitschriften auch eine wesentliche Basis für Veränderungen im Bereich der Struktur wissenschaftlicher Kontroversen.

Die älteren wissenschaftlichen und religionspolitischen Kontroversen der Frühen Neuzeit beruhen auf dem Austausch mehr oder weniger umfänglicher Streitschriften. Dieses Modell wird im 17. und 18. Jahrhundert zunehmend abgelöst von einem Kontroversenmodell, das eine Mehrzahl von intertextuell verknüpften Beiträgen umfasst, die zum Teil unterschiedlichen Darstellungsformen zuzuordnen sind.

Ein Beispiel dafür ist der deutsche Zweig der Phlogiston-Kontroverse, der sich in den Jahren 1786 ff. vor allem in den Zeitschriften entfaltete. Diese Auseinandersetzung ist gekennzeichnet durch ein Mehr-Autoren-Netzwerk, deren deutschsprachige oder ins Deutsche übersetzte Beiträge ein fortlaufend anwachsendes Netz von Text/Text-Zusammenhängen bilden.

Die veränderte Kontroversen-Dynamik ist gekennzeichnet vor allem durch Mehr-Parteien-Konstellationen, die Nutzung einer Vielfalt von etablierten und auch von ad-hoc-Darstellungsformen sowie von vielfältigeren intertextuellen Bezügen, die über den direkten Bezug zu einem Gegner hinausgehen. 
Manche Gesichtspunkte unserer Analysen hierzu ließen sich übersetzen in bzw. beziehen sich auf die Bestimmungsstücke des Praktiken-Begriffs. Darin läge aber noch kein eigener theoretischer Gewinn. Man kann an dieser Stelle andererseits davon ausgehen, dass eine stärkere Berücksichtigung des Zusammenhangs von textuell-kommunikativen Praktiken und fachwissenschaftlichen Praktiken, z.B. Formen und Möglichkeiten des chemischen Experimentierens, durchaus fruchtbar wären. Eine solche Erweiterung der textuellen Forschung würde allerdings die Beteiligung von Fachleuten der jeweiligen Gegenstandsbereiche, z.B. aus der Chemiegeschichte, voraussetzen.

\subsection{Mikrofotografien in der Medizin-Kommunikation: Robert Koch fotografiert erstmals Bakterien ...}

Robert Koch (1843-1910) gehört zu den wichtigsten Pionieren der frühen Bakteriologie. Im Hinblick auf die Identifikation und die genaue Beschreibung von Erregern spielten mehrere technische Verfahren zusammen, v.a. die Nutzung von Färbe-, Isolierungs- und Konservierungsmethoden sowie die Mikrofotografie, deren Zusammenspiel Koch in einem eigenen Beitrag diskutiert (1877). Sogenannte „Photogramme nach der Natur“ schufen damals eine veränderte und verbesserte Argumentations-Grundlage in der damals jungen Bakteriologie, sie ermöglichten die Visualisierung und damit die Überprüfbarkeit von experimentellen Befunden bei der Untersuchung von Gewebe und von Kulturen.

Techno-Bilder dieser Art prägen inzwischen weite Teile der medizinischnaturwissenschaftlichen Kommunikation der Moderne. Sie sind auf das engste verbunden mit der Frage nach dem epistemischen Status von theoretischen Gegenständen, der Frage nach der Rolle von Visualisierungen in den Wissenschaften und auch mit der Frage nach den medial-technischen Grundlagen von Darstellungsformen und Darstellungsmitteln in den Wissenschaften in ihrer historischen Entwicklung.

Man kann von all diesen Möglichkeiten, Handlungsweisen und kommunikativen Verfahren natürlich als von „Praktiken (der Bildverwendung)“ sprechen, aber der Begriff trägt nicht mehr zur Konzeptualisierung dieses spezifischen Bereichs bei als der Begriff „Verfahren“. Wenn man die Bestimmungsstücke des PraktikenBegriffs nutzt, um spezifische Ausprägungen von Parametern wie z.B. „(Mediale) Realisierungsbedingungen“ zu beschreiben, wird man wieder bei einer spezielleren Theoriekomponente landen, die im besten Fall wesentliche Errungenschaften früherer Konzeptionen integriert, die aber in vielen Aspekten nicht vom allgemeinen Praktiken-Begriff gespeist wird, sondern von den spezifischen Erfordernissen des Gegenstandes und von bereits vorhandenen Theoriekomponenten. 


\subsection{Die Multimodalisierung wissenschaftlicher Darstellungsformen}

Betrachten wir ein modernes Lehrbuch, z.B. das Biologie-Lehrbuch von Campbell/Reece (2011), dann sehen wir eine sehr vielgestaltige Form der Nutzung multimodaler Ressourcen und ihres Zusammenspiels. Zu den genutzten Ressourcen gehören u.a. Farbe, z.B. im Rahmen von Farbleitsystemen, Typografie, räumliches Arrangement, die klassische Ressource Text, die Nutzung ganz unterschiedlicher Arten von Abbildungen in Verbindung mit Texten, Formen der Koordination von Text und Bild mit Hilfe von Siglen, die Nutzung von Icons, Linien, farbigen Flächen und anderen Ressourcen.

Auch wenn die koordinierte Nutzung multimodaler Ressourcen seit vielen Jahrhunderten verfügbar ist, ${ }^{6}$ kann man gleichwohl sagen, dass die systematische und großflächige Nutzung multimodaler Kommunikationsangebote in den Wissenschaften seit dem 20. Jahrhundert stetig zunimmt und dass dabei auch deutliche Unterschiede in den verschiedenen Wissenschaftsdomänen zu erkennen sind. Die geisteswissenschaftliche Monografie ist zum Beispiel auch heute noch textlastig und überwiegend linear, während zum Beispiel in medizinischen Lehrbüchern schon früh mit Formen der Text/Bild-Koordination, mit Farbdruck, mit unterschiedlichen Spielarten der Typografie, mit Raumarrangements gearbeitet wurde, weil es dafür einen sinnvollen kommunikativen Einsatz gab.

Auch dieser Befund verdeutlicht, dass man die zu einer Zeit verfügbaren technisch-medialen Potenziale unterscheiden muss von den tatsächlichen Nutzungsweisen und Nutzungsfrequenzen, die zu einer bestimmten Zeit und in einer bestimmten fachlichen Domäne üblich waren. Die Geschichte multimodaler Angebote in den Wissenschaften, ihrer Gestaltungsprinzipien und -spielarten sowie ihrer Nutzung in den Teildisziplinen ist noch nicht geschrieben. Soweit aber aktuell zu sehen, kommen wesentliche Anregungen zur Erforschung textbasierter multimodaler Angebote nicht aus der Praktiken-Diskussion, sondern etwa in den im Literaturverzeichnis aufgeführten Arbeiten von Bateman, Bucher, Kress oder Steinseifer wiederum aus spezifischeren Diskussionszusammenhängen wie z.B. der Text/Bild-Theorie, der Theorie der kommunikativen Verständigung, der Theorie der Medienkommunikation usw.

6 Vgl. etwa Gloning (i.Ersch.) zu Leonhart Thurneyssers Buch über die Erdgewächse (1578). 


\subsection{Digitale Ressourcen und ihre kommunikative Nutzung in den Wissenschaften}

Mit der Verfügbarkeit von Digitalmedien und vor allem mit der zunehmenden Nutzung des Internets für kommunikative Zwecke hat sich das Gefüge der Interaktionsformen und ihrer medialen Grundlagen in den Wissenschaften nachhaltig verändert. In den folgenden Abschnitten möchte ich drei Beispiele geben für neue digitale Formate und für ihre kommunikative Nutzung in den Wissenschaften.

\subsubsection{Mailinglisten: Formen der Nutzung und Entwicklungen}

Mailinglisten gehören zu den medialen Formaten, die relativ früh in unterschiedlichen wissenschaftlichen Teildisziplinen genutzt wurden. Im Rahmen des GieBener Projekts „Interactive Science“ haben wir auch eine Studie zur Nutzung von Mailinglisten in unterschiedlichen Fachzonen durchgeführt, deren Resultate in einem online zugänglichen Open-Access-Band - auch dies eine neue digital gestützte Möglichkeit - dokumentiert sind (Bader et al. 2011).

Mailinglisten gibt es verstärkt seit den 1990er Jahren. Sie weisen fachlichthematisch ein breites Spektrum auf. Unser Korpus umfasste z.B. die B-Greek-, die Shakesper-, die Luhmann-, die Ansax- und viele andere Mailinglisten. Das funktionale Profil dieser Listen bewegte sich im Rahmen von drei Schwerpunkten, die jeweils in unterschiedlichen Gewichtungen vertreten waren: zum einen Information und Service (z.B. Calls for Papers, Stellenausschreibungen), sodann Formen der Kollaboration (z.B. Bitten um Literaturhinweise) und schließlich Formen der Kritik und der Diskussion.

Für den dritten großen Funktionsbereich, Kritik und Kontroverse, haben wir im Gießener Projekt unter anderem eine Kontroverse auf der Linguist-Liste untersucht, deren Ursprung auf eine Rezension zurückgeht und die sehr schnell wesentliche Grundsatzfragen der Grammatikschreibung berührte (Fritz 2011a, S. 198 ff.; Fritz/Gloning 2012). In der Mukherjee-vs.-Huddleston/Pullum-Kontroverse aus dem Jahr 2002 ging es um zwei Hauptkritikpunkte an einer neuen Grammatik des Englischen: die Wahl nur eines theoretischen Modells und die fehlende Korpus-Basierung. Die Rezension ist Ausgangspunkt einer Kontroverse, in der unterschiedliche Verfahren der Kritik und der Verteidigung angewendet wurden.

Als Ergebnis der Kontroverse, die sich in nur wenigen Wochen ereignete, kann man festhalten: (i) Die Kontroverse erlaubte eine schnelle Klärung unterschiedlicher Positionen und Sichtweisen (auch wenn keine „Einigung” erzielt wird). (ii) Die Kontroverse eröffnet den Verfechtern eines Standpunkts ,attention 
space“ für ihre Auffassungen. (iii) Die Kontroverse ermöglicht es, wesentliche Grundsatzfragen in der Grammatikschreibung zu thematisieren; (iv) Auseinandersetzungen dieser Art haben auch einen besonderen Nutzen für Newcomer im Feld: Sie können schnell unterschiedliche Positionen, ihre Verfechter und auch Argumente pro und contra kennenlernen. Besonders hervorzuheben sind die Geschwindigkeit und auch die Reichweite der Auseinandersetzung auf einer Liste, der mehrere Tausend Sprachwissenschaftler/innen auf der ganzen Welt angehören.

Immer wieder beobachtet man auf Mailinglisten auch Versuche, durch Veränderungen an interaktionalen Parametern mit neuen Formen der Formatnutzung zu experimentieren. Ein Beispiel dafür ist das sogenannte RoundTableFormat, das auf der Shakesper-Liste im Jahr 2006 entwickelt und erprobt wurde (dazu Bader/Fritz 2011, S. 67-71). Ausgangspunkt war das Bedürfnis, den Diskussionen auf der Liste für bestimmte Fragen eine strengere und systematischere Form zu geben, das der Moderator der Liste formuliert:

The idea of these RoundTables is to provide a forum for members to use the Internet in such a manner as to enable academic discourse in an alternative platform to conferences, journals, and such. Thus, RoundTable discussions will be conducted on a more formal level than ordinary list discussions: contributors will be expected to reflect upon their offerings [...] before submitting them and to be knowledgeable of the subject under discussion. (Hardy M. Cook, 9.12.2006)

In der Umsetzung wird das technisch-mediale Format neuartig genutzt, indem neue „Spielregeln“ eingeführt und bestimmte interaktionale Parameter festgeschrieben werden. So gibt es etwa in der Rollenkonstellation neue Vorgaben für einen Gastmoderator und Qualitätsvorgaben für die Vorbereitung der Teilnehmer. Die Untersuchungen, auf die ich mich hier beziehe, sind alle auf die kommunikative Praxis in den Wissenschaften und ihre Entwicklung bezogen, aber ohne expliziten Bezug zu einem Praktiken-Konzept.

Im Hinblick auf das Praktiken-Konzept ist dabei besonders wichtig: Wir blicken hier gleichsam ins Laboratorium der kommunikativen Neuerung, des Experimentierens mit neuen medialen Möglichkeiten, der zeitweisen Verfestigung aber auch der Aufgabe von kommunikativen Optionen. Handlungstheoretische Konzeptionen stützen sich für die Modellierung solcher Entwicklungen vor allem auf Invisible-Hand-Modelle, in denen auch Innovationen und kommunikativen „Eintagsfliegen“ der gebührende Raum gewährt wird. Historischdynamische Denkfiguren im Rahmen der Praktiken-Theorie beziehen sich vielfach auf ähnliche Traditionen, allerdings scheint mir, dass dort eher der Gesichtspunkt der Reproduktion und der Variation bereits vorhandener Praktiken im Vordergrund steht. 
Die Mailinglisten-Befunde werfen dagegen ein etwas anderes Licht auf die Auffassung, dass Praktiken ,emergente“ Produkte wiederholten, routinierten Handelns sind. Unsere Beispiele zeigen, dass einzelne Handlungsweisen bzw. kommunikative Nutzungsweisen medialer Formate intentional konstruiert wurden mit spezifischen kommunikativen Zielsetzungen und in Kenntnis der Variationsparameter und der kommunikativen Folgen ihrer Veränderung. ${ }^{7}$

Wenn Praktiken als „soziale Strukturen“, als „sozial konsentierte Routinen, die sedimentiert sind“ gefasst werden, dann fällt eigentlich der sprachhistorisch und in evolutionärer Hinsicht besonders interessante Bereich der Innovationen, der kommunikativen Versuche, der Eintagsfliegen und der kommunikativen Holzwege aus der Betrachtung heraus. ${ }^{8}$ Die kommunikative Mechanik der Innovation und der Variation mit all ihren Spielarten muss aber ein zentraler Bestandteil der Lehre vom etablierten Sprachgebrauch und seiner Entstehung sein, in welcher Umgebung auch immer.

\subsubsection{Qualitätssicherung und Kritik im Open Peer Review und Möglichkeiten des Kommunikationsdesign}

Qualitätssicherung und Kritik gehören zu den grundlegenden Aufgaben der Wissenschaftskommunikation. Im Rahmen der digitalen Wissenschaftskommunikation sind auch in diesem Funktionsbereich neue Interaktionsweisen eingerichtet worden. Ein gutes Beispiel hierfür ist das Open Peer Review-Verfahren der digitalen Open Access-Zeitschrift „Atmospheric Chemistry and Physics“ (ACP), das Gerd Fritz in einem Beitrag zum Band „Digitale Wissenschaftskommunikation“ untersucht hat (Fritz 2011b; siehe Abb. 2).

Die Besonderheiten dieses Begutachtungs- und Diskussionsverfahrens bestehen darin, dass neben zwei anonymen Gutachter/innen auch sogenannte „Short comments“ von Wissenschaftler/innen der Gemeinschaft sowie Author comments möglich und erwünscht sind, die Diskussion findet öffentlich statt, die Gutachten und alle anderen Beiträge sind also jederzeit zugänglich, schließlich ist auch der Diskussions- und Qualitätssicherungsbereich (ACPD) zitierbar, alle Beiträge bleiben langfristig verfügbar und durch stabile Adressen ansteuerbar. Auch wenn ein Manuskript nicht für die Veröffentlichung in ACP akzeptiert wird, so bleiben doch die eingereichten und diskutierten Versionen verfügbar, auch die Stellungnahmen bleiben verfügbar und zitierbar.

7 Vgl. hierzu auch den Beitrag von Nyre (2014).

8 Vgl. zu dieser Perspektive auch Gloning (2013). 


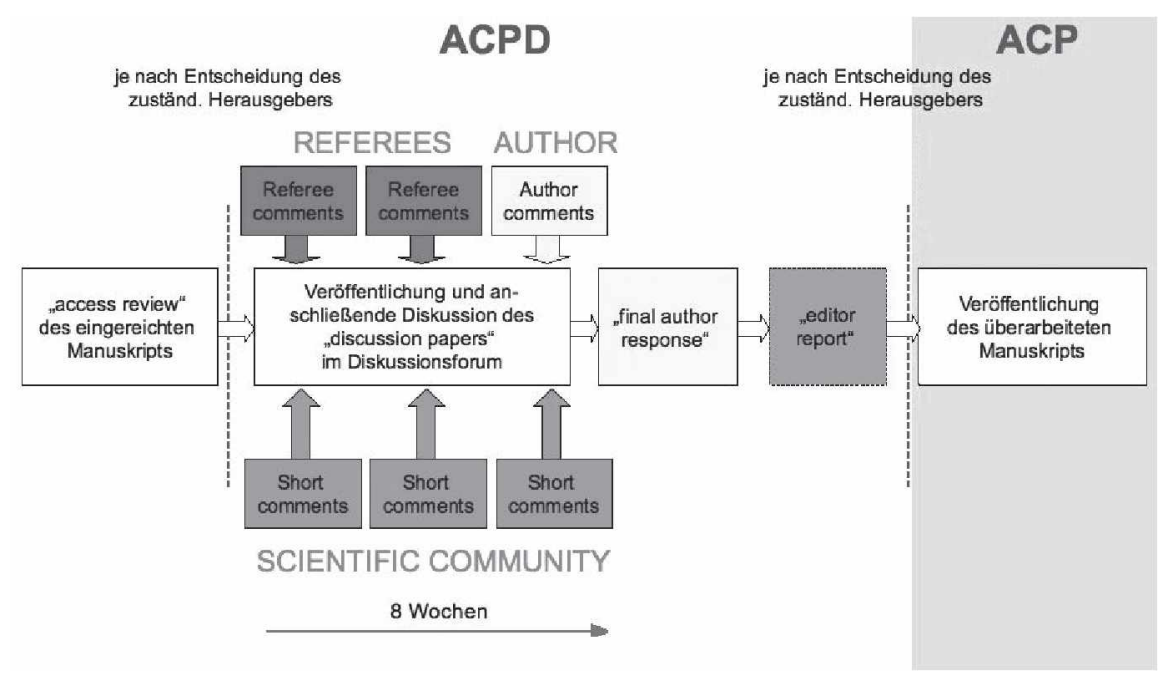

Abb. 2: Open-Peer-Review-Verfahren von ACP; Quelle: Fritz (2011b, S. 149), vgl. Pöschl (2010)

Am Beispiel der Makarieva-Kontroverse, in der es um grundlegende Fragen der Theorie der Hurrikane ging, hat Fritz (2011b) gezeigt, wie in einem vergleichsweise kurzen Zeitraum von acht Wochen die weitreichende These, dass die etablierte Theorie der Hurrikane den Gesetzen der Thermodynamik widerspricht, im digitalen ACPD-System intensiv diskutiert wurde (vgl. auch Fritz/Gloning 2012, S. 219 ff.). An der Diskussion beteiligen sich die Autor/innen, drei Gutachter, vier weitere Teilnehmer und der Herausgeber mit einem breiten Spektrum von kommunikativen Zügen, so zum Beispiel mit Einwänden, die sich auf die physikalischen Grundlagen des Manuskripts beziehen („paper contains bad physics“).

Im Hinblick auf das Praktiken-Konzept bietet diese Teilbeschreibung des Open Peer Review in „Atmospheric Chemistry and Physics“ ein weiteres Beispiel für die intentionale Konstruktion von kommunikativen Verfahren, die wohl auf älteren Schemata aufsitzen, aber in wesentlichen Punkten modifiziert sind.

Wenn man die Perspektive weiterführt und voraussetzt, dass das Ziel des Open-Peer-Review-Prozesses neben der Entscheidung über die Annahme zur Publikation vor allem auch die Optimierung eingereichter Manuskripte darstellt, dann kann man als weiteren Ausgangspunkt die Frage stellen, wie sich dieses Optimierungsverfahren noch weiter optimieren ließe.

Ein erster Vorschlag könnte darin bestehen, ein thematisches Schema für Reviewer einzuführen, in dem eine Batterie von Beurteilungsaspekten und -kriterien aufgeführt ist, die ein Gutachter daraufhin überprüfen kann, ob sie für die Beurteilung eines bestimmten Manuskripts relevant sind. 
Ein weiterer Parameter ist die Frage nach dem Zeitpunkt der Veröffentlichung der Gutachten. In der gegenwärtigen Praxis wird ein Gutachten veröffentlicht, sobald es vorliegt. Der spätere Gutachter kennt also Tenor und Inhalt seines Vorgängers. Man kann sich nun fragen, ob es nicht sinnvoller wäre, die Gutachten erst dann zu veröffentlichen, wenn alle Gutachten vorliegen. Diese Veränderung könnte Folgen haben vor allem für die Unabhängigkeit der Beurteilung, aber auch für die eigenständige Entwicklung von kritischer Substanz.

Im Hinblick auf die thematische Portionierung und die Kennzeichnung von kritischen Punkten kann man von den frühneuzeitlichen Streitschriften lernen. Dort war es üblich, dass die Beiträger die kritische Substanz der Kontroverse jeweils in „Punkte“ organisierten, die klar abgrenzbar und über Formen der Nummerierung auch ansprechbar waren. Es wäre technisch nicht schwer, ein digitales Gegenstück für diese frühneuzeitliche Form der Buchführung über kritische „Punkte“ und den jeweiligen Stand ihrer Beantwortung einzurichten. Man kann also beim Verfahren der systematischen Variation interaktionaler Parameter auch historische Praktiken als Anregung nutzen.

Sowohl die Betrachtung des ACP-Peer-Review-Verfahrens als auch meine Überlegungen zu Formen von „Kommunikationsdesign“, bei dem interaktionale Parameter gezielt und in Anlehnung an historische Praktiken im Hinblick auf kommunikative Ziele und Aufgaben verändert werden, zeigen, dass neue Handlungsmöglichkeiten (Praktiken) auch geplant und ggf. intentional installiert werden können. Sie sind dann nicht das ungeplante Resultat einer kollektiven sozialen Praxis. Gleichwohl müssen sich auch geplante kommunikative Neuerungen in der Praxis bewähren und ggf. etablieren.

Die hier besprochenen Verfahren des digitalen Open Peer Review und auch viele andere digital gestützte Formate werfen weiterhin die Frage nach der Lokalitätsthese und nach dem Raumbezug von Praktiken auf, wie sie u.a. von Pennycook (2010) und von Giddens (1995) vorgebracht wurden. In den meisten Fällen weiß man nicht, wo ein digitales Angebot ausgearbeitet und aufgesetzt wurde. Und man muss es auch nicht wissen. Es ist auch nicht wichtig, wo die Server stehen und welche Wege der Datenfluss nimmt. Ist es erheblich, von welchem Ort aus Mails oder andere digitale Beiträge etwa zu einem Open-Peer-ReviewVerfahren abgeschickt wurden und an welchem Ort sie gelesen werden? Ich meine nicht.

Wenn man sich hier in die Redeweise von einem digitalen Raum flüchten wollte, dann wäre es nicht mehr der ursprüngliche Raumbezug mit seinen Bezügen zur Körperlichkeit, sondern eine Form der Metaphorik, die tiefgreifende Unterschiede etwa zu Formen der leiblichen Kopräsenz in Gesprächen verdecken würde. Das Lokale, der Raumbezug, auch das Körperlich-Leibliche verdient in der Praktikendiskussion im Licht rasanter medialer Entwicklungen eine Neubestimmung. 


\subsubsection{Digitale Sprachdaten und digitale Werkzeuge für die Forschung}

In den beiden vorhergehenden Abschnitten wurden exemplarisch mediale Formate und kommunikative Nutzungsweisen vorgestellt, die im Rahmen der Wissenschaftskommunikation instrumentell eingesetzt werden. Daneben gibt es aber auch ein weiteres Resultat, das als Konsequenz der Digitalisierung aufzufassen ist, die zunehmende Nutzung von digitalen Sprachdaten und von darauf bezogenen Werkzeugen, bei denen Sprache nicht das Mittel, sondern der Gegenstand der Forschung ist. Im Unterschied zur evolutionären Entwicklung von Interaktionsformen des Alltags werden - wie im Abschnitt 3.7.2 schon angedeutet - auch solche technisch-medialen Forschungsarrangements in der Korpuslinguistik oder in vielen Bereichen der Digital Humanities gezielt entworfen und hergestellt.

Als ein Beispiel dafür, wie eine neue medial-technische Infrastruktur auch den Status und die Natur des Forschungsprozesses verändern kann, sei hier das Projekt Reproducible Research/Open Science genannt, dem sich z.B. das „Potsdam Mind Research Repository“ zuordnet. Die Grundidee ist es, neben den Artikeln mit wissenschaftlichen Resultaten auch die zugrundeliegenden Forschungsdaten inklusive der digitalen Auswertungsprogramme und -routinen öffentlich zur Verfügung zu stellen, zu dokumentieren und Forschung damit dauerhaft reproduzierbar und ggf. auch modifizierbar zu machen:

\footnotetext{
Seite: 36

The Potsdam Mind Research Repository (PMR2) provides access to peer-reviewed publications along with data and scripts for analyses and figures reported in them. We refer to these units as „paper packages“. We hope to achieve the following goals:

- Document data and analyses used in our publications in a public forum.

- Invite readers (a) to reproduce our analyses/figures, (b) to try out and possibly publish alternative analyses, or (c) to adopt our scripts for their own data.

- Receive feedback about our scripts, both about necessary corrections of errors and more elegant alternative code.

(http://read.psych.uni-potsdam.de/pmr2)
}

Infrastrukturprojekte wie CLARIN-D (Common Language Resources and Technology Infrastructure, Deutschland) zielen darauf, digitale Ressourcen in einer nachhaltigen Web- und Zentren-Infrastruktur bereitzustellen und nach Möglichkeit zu integrieren (http://de.clarin.eu). Auch die gezielte Integration von Sprachdaten und die planmäßige Kombinierbarkeit von digitalen Werkzeugen in aufgabenorientierten Toolchains (z.B. WebLicht) gehört genauso wie z.B. die „federated content search“,

9 www.clarin.eu/content/federated-content-search-clarin-fcs. 
an der auch das Institut für Deutsche Sprache beteiligt ist, zu den Aspekten der Entwicklung neuer Formate auf digitaler Grundlage.

Im Lichte einer Praxisperspektive kann man sagen, dass die Werkzeugentwicklung im Bereich der eHumanities eine Art von Praxis ist, die neue Praktiken erarbeiten und auf wissenschaftliche Zielsetzungen beziehen soll. Man könnte hier vielleicht von einer Praktiken-Generierungs-Praxis sprechen. Viele Projekte im Bereich der eHumanities-Forschung dienen im Kern auch diesem Zweck. Die Frage, wie die Konstruktionsarbeiten an neuen digital gestützten Handlungsmöglichkeiten bzw. Praktiken im wissenschaftlichen Alltag tatsächlich verlaufen, ist eine eigene Aufgabe für die Ethnografen dieses Handlungsbereiches.

\section{Zusammenfassung}

Im vorliegenden Beitrag diskutiere ich von einem handlungstheoretischen Standpunkt aus, der auch sprachhistorisch-evolutionäre und mediale Erweiterungen umfasst, Vorschläge zur Rolle des Praktiken-Begriffs bzw. (im weiteren Sinne) des Praktiken-Konzepts. In einem ersten Schritt formuliere ich sieben eher kritische Thesen zu einem Ausschnitt der Praktiken-Literatur, den ich studiert habe, suche dabei auch nach Konsens und Dissens. In einem zweiten Schritt stelle ich Befunde zu ausgewählten Veränderungen in der Wissenschaftskommunikation seit der Erfindung des Buchdrucks bis hin zu aktuellen Entwicklungen im Zeichen der Digitalisierung dar und frage dabei, ob die bisher verfügbaren, handlungstheoretisch orientierten Beschreibungen durch die Anwendung des PraktikenBegriffs gewonnen hätten - eine Frage, die in weiten Teilen verneint werden muss.

Leitperspektiven der Diskussion waren u.a. die Unterscheidung des Praktiken-Begriffs von einer allgemeineren Praxis-Perspektive, die Frage nach der wissenschaftsgeschichtlichen Tradition und der Neuheit von Aspekten der Praktiken-Diskussion für die sprachwissenschaftliche Forschung, die Frage nach dem Format einer integrierten sprachgebrauchsorientierten Konzeption und auch ausgewählte Aspekte des zeitlich und disziplinär differenzierten Verlaufs der praxis- bzw. praktiken-orientierten Diskussion.

Das Resultat aus beiden Teilen ist eine gewisse Skepsis, ob der PraktikenBegriff die Vielfalt der bisherigen pragmatischen Konzepte und Werkzeuge integrieren kann, ob seine Anwendung den vielfältigen aktuellen und historischen Kommunikationsbereichen und auch den darauf bezogenen Forschungstraditionen und neuen Aufgaben gerecht wird. 


\section{Literatur}

Austin, John L. (1956/1957): A plea for excuses. In: Proceedings of the Aristotelian Society 57, S. 1-30.

Bader, Anita et al. (2011): Vom Überleben einer bedrohten Spezies. Untersuchungen zur Entwicklung der Nutzung wissenschaftlicher Mailinglists. In: Gloning/Fritz (Hg.), S. 87-116.

Bader, Anita/Fritz, Gerd (2011): Zur Entwicklung von Formaten und Kommunikationsformen in der digitalen Wissenschaftskommunikation - eine evolutionäre Betrachtungsweise. In: Gloning/Fritz (Hg.), S. 55-86.

Bateman, John A. (2008): Multimodality and genre. A foundation for the systematic analysis of multimodal documents. Basingstoke.

Bauman, Richard/Sherzer, Joel (Hg.) (1974): Explorations in the ethnography of speaking. London/New York.

Bourdieu, Pierre (2012): Entwurf einer Theorie der Praxis: Auf der ethnologischen Grundlage der kabylischen Gesellschaft. 3. Aufl. Frankfurt a.M. [Frz. Erstveröffentl. Genf 1972.]

Bucher, Hans-Jürgen (1999): Sprachwissenschaftliche Methoden der Medienforschung. In: Leonhard, Joachim-Felix (Hg.): Medienwissenschaft. Ein Handbuch zur Entwicklung der Medien und Kommunikationsformen. 1. Halbbd. (= Handbücher zur Sprach- und Kommunikationswissenschaft 15.1). Berlin/New York, S. 213-231.

Bucher, Hans-Jürgen (2000): Formulieren oder Visualisieren? Multimodalität in der Medienkommunikation. In: Richter, Gerd/Riecke, Jörg/Schuster, Britt M. (Hg.): Raum, Zeit, Medium Sprache und ihre Determinanten. Festschrift für Hans Ramge zum 60. Geburtstag. (= Arbeiten der Hessischen Historischen Kommission 20). Darmstadt, S. 661-691.

Bucher, Hans-Jürgen (2007): Textdesign und Multimodalität. Zur Semantik und Pragmatik medialer Gestaltungsformen: In: Roth, Kersten Sven/Spitzmüller, Jürgen (Hg.): Textdesign und Textwirkung in der massenmedialen Kommunikation. Konstanz, S. 49-76.

Bucher, Hans-Jürgen (2010): Multimodalität - eine Universalie des Medienwandels: Problemstellungen und Theorien der Multimodalitätsforschung. In: Bucher/Gloning/Lehnen ( $\mathrm{Hg}$.), S. 41-79.

Bucher, Hans-Jürgen/Gloning, Thomas/Lehnen, Katrin (Hg.) (2010): Neue Medien - neue Formate. Ausdifferenzierung und Konvergenz in der Medienkommunikation. (=Interaktiva 10). Frankfurt a.M./New York.

Campbell, Neil A./Reece, Jane B. (2011): Biologie. 8., aktual. Aufl. München.

Cohen, Marcel (1956): Pour une sociologie du langage. Paris.

Coseriu, Eugenio (1958): Sincronía, diacronía e historia. El problema del cambio lingüístico. Montevideo. [Dt. Ausg.: Synchronie, Diachronie und Geschichte. München 1974.]

Deppermann, Arnulf (2007): Grammatik und Semantik aus gesprächsanalytischer Sicht. (= Linguistik - Impulse \& Tendenzen 14). Berlin/New York.

Everett, Daniel (2005): Cultural constraints on grammar and cognition in Piraha. Another look at the design features of human language. In: Current Anthropology 46, S. 621-646.

Everett, Daniel (2012): Language: The cultural tool. London.

Feilke, Helmuth (1996): Sprache als soziale Gestalt. Ausdruck, Prägung und die Ordnung der sprachlichen Typik. Frankfurt a.M.

Fiehler, Reinhard (2003): Was sind Grundeinheiten gesprochener Sprache? Ein altes Problem und ein neuer Lösungsvorschlag. In: Sprachtheorie und germanistische Linguistik 13, S. $145-172$. 
Fiehler, Reinhard et al. (2004): Eigenschaften gesprochener Sprache. (= Studien zur Deutschen Sprache 30). Tübingen.

Frake, Charles O. (1961): The diagnosis of disease among the Subanum of Mindanao. In: American Anthropologist 63, S. 113-132.

Frake, Charles O. (1964): How to ask for a drink in Subanum. In: American Anthropologist 66, 6.2, S. 127-132.

Fritz, Gerd (2010): Controversies. In: Jucker, Andreas/Taavitsainen, Irma (Hg.): Handbooks of pragmatics. Bd. 8: Historical pragmatics. Berlin/New York, S. 451-481.

Fritz, Gerd (2011a): Lehrreiche wissenschaftliche Kontroversen im Internet? In: Gloning/Fritz (Hg.), S. 193-204.

Fritz, Gerd (2011b): Wirbelstürme im digitalen Open-Peer-Review-Verfahren. Die MakarievaKontroverse in Atmospheric Chemistry and Physics (2008/09) - Eine Fallstudie. In: Gloning/Fritz (Hg.), S. 143-174.

Fritz, Gerd (2013): Dynamische Texttheorie. Gießen. Internet: http://geb.uni-giessen.de/geb/ volltexte/2013/9243/ (Stand: 15.9.2015).

Fritz, Gerd/Bader, Anita (2010): Digitale Formate in der Wissenschaftskommunikation. Konstellationen und Konvergenzen. In: Bucher/Gloning/Lehnen (Hg.), S. 337-355.

Fritz, Gerd/Gloning, Thomas (2012): Critique and controversy in digital scientific communication. New formats and their affordances. In: Van Eemeren, Frans H./Garssen, Bart (Hg.): Exploring argumentative contexts. (= Argumentation in Context 4). Amsterdam/Philadelphia, S. 213-231.

Giddens, Anthony (1995): Die Konstitution der Gesellschaft. Grundzüge einer Theorie der Strukturierung. (= Theorie und Gesellschaft 1). Frankfurt a.M.

Giesecke, Michael (1991): Der Buchdruck in der frühen Neuzeit: Eine historische Fallstudie über die Durchsetzung neuer Informations- und Kommunikationstechnologien. Frankfurt a.M.

Gloning, Thomas (1996): Bedeutung, Gebrauch und sprachliche Handlung. Ansätze und Probleme einer handlungstheoretischen Semantik aus linguistischer Sicht. (= Reihe Germanistische Linguistik 170). Tübingen.

Gloning, Thomas (1999): The pragmatic form of religious controversies around 1600 . A case study in the Osiander vs. Scherer \& Rosenbusch controversy. In: Jucker, Andreas H./Fritz, Gerd/Lebsanft, Franz (Hg.): Historical dialogue analysis. (= Pragmatics \& Beyond 66). Amsterdam/Philadelphia, S. 81-110.

Gloning, Thomas (2008): „Man schlürft Schaupielkunst...“. Spielarten der Theaterkritik. In: Hagestedt, Lutz (Hg.): Literatur als Lust. Begegnungen zwischen Poesie und Wissenschaft. Festschrift für Thomas Anz zum 60. Geburtstag. (= Reihe Theorie und Praxis der Interpretation 6). München, S. 59-86.

Gloning, Thomas (2013): Historischer Wortgebrauch und Themengeschichte: Grundfragen, Corpora, Dokumentationsformen. In: Hafemann, Ingelore (Hg.): Perspektiven einer corpusbasierten historischen Linguistik und Philologie. Berlin, S. 317-370. Internet: http://edoc. bbaw.de/volltexte/2013/2448 (Stand: 15.9.2015).

Gloning, Thomas (i.Ersch.): Textkomposition und Multimodalität in Thurneyssers Buch über die Erdgewächse (1578). Eine Erkundung. In: Schuster, Britt-Marie/Dogaru, Dana (Hg.): Wirksame Rede im Frühneuhochdeutschen. Syntaktische und textstilistische Aspekte. Festschrift für Monika Rössing-Hager. Frankfurt a.M.

Gloning, Thomas/Fritz, Gerd (Hg.) (2011): Digitale Wissenschaftskommunikation. Formate und ihre Nutzung. Gießen. Internet: http://geb.uni-giessen.de/geb/volltexte/2011/8227 (Stand: 15.9.2015). 
Gren, Friedrich Albrecht Carl (Hg.) (1790): Journal der Physik. Jahr 1790. Erster Band. Halle/ Leipzig.

Grice, Herbert P. (1989): Studies in the way of words. Cambridge, MA/London.

Gross, Alan G./Harmon, Joseph E./Reidy, Michael (2002): Communicating science. The scientific article from the 17th century to the present. Oxford.

Habel, Thomas (2007): Gelehrte Journale und Zeitungen der Aufklärung: Zur Entstehung, Entwicklung und Erschliessung deutschsprachiger Rezensionszeitschriften des 18. Jahrhunderts. (= Presse und Geschichte 17). Bremen.

Hanks, William F. (1987): Discourse genres in a theory of practice. In: American Anthropologist 14, S. 668-692.

Hanks, William F. (1996): Language \& communicative practices. (= Critical Essays in Anthropology). Oxford/Boulder.

Heringer, Hans Jürgen (1999): Das höchste der Gefühle. Empirische Studien zur distributiven Semantik. (= Stauffenburg Linguistik 15). Tübingen.

Heringer, Hans Jürgen et al. (1977): Einführung in die Praktische Semantik. Heidelberg.

Heritage, John (2011): Conversation analysis: Practices and methods. In: Silverman, David (Hg.): Qualitative research. Issues of theory, method and practice. 3. Aufl. Los Angeles, S. 208230.

Hymes, Dell (Hg.) (1964): Language in culture and society. A reader in linguistics and anthropology. New York/Evanston/London.

Keller, Rudi (1990): Sprachwandel. Von der unsichtbaren Hand in der Sprache. Tübingen.

Keller, Rudi (1995): Zeichentheorie. Zu einer Theorie semiotischen Wissens. Tübingen/Basel.

Koch, Robert (1877): Untersuchungen über Bacterien. VI. Verfahren zur Untersuchung, zum Conserviren und Photographiren der Bacterien. In: Cohn, Ferdinand (Hg.): Beiträge zur Biologie der Pflanzen. Bd. 2. Breslau, S. 399-434. Tafeln Photogramme im Lichtdruck XIV, $\mathrm{XV}, \mathrm{XVI}$.

Kress, Gunther (2009): What is mode? In: Jewitt, Carey (Hg.): The Routledge handbook of multimodal analysis. London/New York, S. 54-67.

Labov, William (1972): Rules for ritual insults. In: Sudnow, David (Hg.): Studies in social interaction. New York, S. 120-169.

Linke, Angelika (2011): Signifikante Muster - Perspektiven einer kulturanalytischen Linguistik. In: Wâghäll Nivre, Elisabeth et al. (Hg.): Begegnungen. Das 8. Nordisch-Baltische Germanistentreffen in Sigtuna, 11.-13.6.2009. (= Stockholmer germanistische Forschungen 74). Stockholm, S. 23-44.

Malinowski, Bronislaw (1923): The problem of meaning in primitive languages. In: Ogden, Charles K./Richards, Ivor A.: The meaning of meaning: A study of the influence of language upon thought and of the science of symbolism. New York, S. 296-336.

Malinowski, Bronislaw (1984): Argonauten des westlichen Pazifik. Ein Bericht über Unternehmungen und Abenteuer der Eingeborenen in den Inselwelten von Melanesisch-Neuguinea. Frankfurt a.M. [Engl. Erstveröffentlichung London 1922.]

Mondada, Lorenza/Schmitt, Reinhold (Hg.) (2010): Situationseröffnungen. Zur multimodalen Herstellung fokussierter Interaktion. (= Studien zur Deutschen Sprache 47). Tübingen.

Muckenhaupt, Manfred (1986): Text und Bild. Grundfragen der Beschreibung von Text-BildKommunikationen aus sprachwissenschaftlicher Sicht. (= Tübinger Beiträge zur Linguistik 271). Tübingen. 
Muckenhaupt, Manfred (1999): Die Grundlagen der kommunikationsanalytischen Medienwissenschaft. In: Medienwissenschaft. In: Leonhard, Joachim-Felix (Hg.): Medienwissenschaft. Ein Handbuch zur Entwicklung der Medien und Kommunikationsformen. 1. Halbbd. (= Handbücher zur Sprach- und Kommunikationswissenschaft 15). Berlin/New York, S. 28-57.

Niemann, Robert (2015): Attributive Junktion in der Wissenschaftssprache. Eine praxistheoretische Betrachtung. In: Hennig, Mathilde (Hg.): Junktion in der Attribution: Ein Komplexitätsphänomen aus grammatischer, psycholinguistischer und praxistheoretischer Perspektive. (= Linguistik - Impulse \& Tendenzen 62). Berlin, S. 239-282.

Nyre, Lars (2014): Media design Method: Combining Media Studies with Design Science to make New Media. In: The Journal of Media Innovations 1, 1, S. 86-109.

Paul, Hermann (1920): Prinzipien der Sprachgeschichte. 5. Aufl. Halle a.d.S. [Erstveröffentl. Halle a.d.S. 1880.]

Pennycook, Alastair (2010): Language as a local practice. London/New York.

Pöschl, Ulrich (2010): Interactive Open Access Publishing and Peer Review: The Effectiveness and Perspectives of Transparency and Self-Regulation in Scientific Communication and Evalutation. In: Liber Quarterly 19, S. 293-314.

Reckwitz, Andreas (2003): Grundelemente einer Theorie sozialer Praktiken. Eine sozialtheoretische Perspektive. In: Zeitschrift für Soziologie 32, S. 282-301.

Schatzki, Theodore R. (1996): Social practices. A Wittgensteinian approach to human activity and the social. Cambridge.

Schatzki, Theodore R. (2001): Introduction. In: Schatzki, Theodore R./Knorr Cetina, Karin/von Savigny, Eike (Hg.): The practice turn in contemporary theory. London, S. 1-14.

Schatzki, Theodore R. (2002): The site of the social. A philosophical account of the constitution of social life and change. University Park, PA.

Schegloff, Emanuel A. (1972): Notes on a conversational practice: Formulating place. In: Sudnow, David (Hg.): Studies in social interaction. New York, S. 325-345.

Schegloff, Emanuel A. (1997): Practices and actions: Boundary cases of other-initiated repair. In: Discourse Processes 23, S. 499-545.

Schlieben-Lange, Brigitte (1983): Traditionen des Sprechens. Elemente einer pragmatischen Sprachgeschichtsschreibung. Stuttgart.

Schmidt, Jürgen Erich (2010): Language and space: The linguistic dynamics approach. In: Auer, Peter/Schmidt, Jürgen Erich (Hg.): Language and space. An international handbook of linguistic variation. 1. Halbbd.: Theories an methods. (= Handbücher zur Sprach- und Kommunikationswissenschaft 30.1). Berlin/New York, S. 201-225.

Schröder, Thomas (2003): Die Handlungsstruktur von Texten. Ein integrativer Beitrag zur Texttheorie. Tübingen.

Statuta Collegii Sapientiae (1497 [1957]). Faksimile, mit einer Einführung hrsg. v. J.H. Beckmann. Lat. Text besorgt und ins Deutsche übers. von R. Feger. Lindau/Konstanz 1957.

Steinseifer, Martin (2011): Die Typologisierung multimodaler Kommunikationsangebote - Am Beispiel der visuellen Aspekte seitenbasierter Dokumente. In: Habscheid, Stephan (Hg.): Textsorten, Handlungsmuster, Oberflächen. Berlin/New York, S. 164-189.

Strecker, Bruno (1987): Strategien des kommunikativen Handelns: Zur Grundlegung einer Grammatik der Kommunikation. (= Sprache der Gegenwart 73). Düsseldorf.

Streeck, Jürgen (2001): Praxeologie. Neue Wege materialistischer Sprachwissenschaft. In: Gruber, Helmut/Menz, Florian (Hg.): Interdisziplinarität in der Angewandten Sprachwissenschaft. Methodenmenü oder Methodensalat? (= Sprache im Kontext 10). Frankfurt a.M., S. 33-56. 
Stukenbrock, Anja (2015): Deixis in der face-to-face-Interaktion. (= Linguae \& Litterae 47). Berlin/München.

Thurneysser zum Tuhrn, Leonhart (1578): Historia Vnnd Beschreibung Influentischer/ Elementischer vnd Natürlicher Wirckungen/ Aller fremden vnnd Heimischen Erdgewechssen/ auch jrer Subtiliteten/ sampt warhafftiger vnd Künstlicher Conterfeitung derselbigen ... Berlin.

Wittgenstein, Ludwig (1984): Werkausgabe. Bd. 1: Tractatus logico-philosophicus, Tagebücher 1914-1916, Philosophische Untersuchungen. Frankfurt a.M.

Ylönen, Sabine (2001): Entwicklung von Textsortenkonventionen: Am Beispiel von Originalarbeiten der Deutschen Medizinischen Wochenschrift (DMW). (= Leipziger FachsprachenStudien 15). Frankfurt a.M. 